\title{
Anti-Inflammatory Effect on Colitis and Modulation of Microbiota by Fermented Plant Extract Supplementation
}

\author{
Manabu Sugimoto ${ }^{1, *(\mathbb{D})}$, Toshiro Watanabe ${ }^{2}$, Motoko Takaoka ${ }^{3}{ }^{\complement}$, Kyoko Suzuki $^{3}$, Tadatoshi Murakami ${ }^{4}$, \\ Nobutada Murakami ${ }^{4}$ and Shoichi Sumikawa ${ }^{4}$ \\ 1 Institute of Plant Science and Resources, Okayama University, 2-20-1 Chuo, Kurashiki, \\ Okayama 710-0046, Japan \\ 2 Department of Food and Nutrition, Sonoda Women's University, 7-29-1 Minamitsukaguchi, Amagasaki, \\ Hyogo 661-8520, Japan; watnb-ts@sonoda-u.ac.jp \\ 3 Department of Biosphere Sciences, Kobe College, 4-1 Okadayama, Nishinomiya, Hyogo 662-8505, Japan; \\ takaoka@mail.kobe-c.ac.jp (M.T.); suzuki@mail.kobe-c.ac.jp (K.S.) \\ 4 Functional Food Creation Research Institute Co., Ltd., 4422-1 Yoshikawa, Kibichuo, Kaga, \\ Okayama 716-1241, Japan; murakami@kinousei.co.jp (T.M.); n-murakami@kinousei.co.jp (N.M.); \\ sumikawa@kinousei.co.jp (S.S.) \\ * Correspondence: manabus@okayama-u.ac.jp; Tel.: +81-86-424-1661
}

check for updates

Citation: Sugimoto, M.; Watanabe, T.; Takaoka, M.; Suzuki, K.; Murakami, T.; Murakami, N.; Sumikawa, S. Anti-Inflammatory Effect on Colitis and Modulation of Microbiota by Fermented Plant Extract Supplementation. Fermentation 2021, 7,55. https://doi.org/10.3390/ fermentation7020055

Academic Editor: Hiroshi Kitagaki

Received: 12 March 2021

Accepted: 3 April 2021

Published: 6 April 2021

Publisher's Note: MDPI stays neutral with regard to jurisdictional claims in published maps and institutional affiliations.

Copyright: (c) 2021 by the authors. Licensee MDPI, Basel, Switzerland. This article is an open access article distributed under the terms and conditions of the Creative Commons Attribution (CC BY) license (https:/ / creativecommons.org/licenses/by/ $4.0 /)$.

\begin{abstract}
Although results of recent studies suggest that fermented foods strongly affect the gut microbiota composition and that they relieve inflammatory bowel disease symptoms, some reports have described that fermented foods increase some inflammation markers based on differences in fermented food materials. This study evaluated the effects of fermented plant extract (FPE) on dextran sulfate sodium (DSS)-induced colitis in mice and the effects on fecal microbiota composition in humans. Mice fed 5\% FPE with 3\% DSS (FPE group) showed no body weight loss, atrophy of colonic length, or bloody stool, similar to mice fed a basal diet (negative group), whereas mice fed 3\% DSS (positive group) exhibited those effects. Concentrations of inflammation markers IL-6 and TNF- $\alpha$ were not significantly different between FPE and negative groups; however, those concentrations became higher in the positive group. 16S ribosomal RNA gene sequencing was used to characterize fecal microbiota in healthy women before and after 3-month FPE supplementation. The FPE supplementation induced increases in Firmicutes phyla and in Clostridiales order, which play a central role in inflammation suppression. These results suggest that FPE enhances Clostridiales growth in the gut and that it has an anti-inflammatory effect.
\end{abstract}

Keywords: fermented plant extract; microbiota; dextran sulfate sodium; inflammatory; Clostridiales

\section{Introduction}

Inflammatory bowel disease, as typified by diseases such as ulcerative colitis and Crohn's disease, affects the gastrointestinal tract. No satisfactory treatment has been found because its causes remain unidentified. Moreover, the Japanese Ministry of Health, Labor, and Welfare has specified these diseases as intractable diseases. The number of patients with these diseases has been increasing in Japan [1,2]. Lack of dietary fiber and the consumption of western foods are implicated as reasons. Actually, dietary changes alter gut microbiota composition. In fact, dysbiosis and disruption of immunological homeostasis have been associated with inflammatory diseases [3-13] because the gut microbiota affects immunologic, nutritional, and metabolic processes of the human body [14], indicating that diet strongly affects inflammatory bowel disease.

Functional foods are part of the human diet. They have been demonstrated to provide health benefits and to decrease the risk of chronic diseases beyond those provided by adequate nutrition [15]. Functional foods include naturally occurring bioactive substances, supplemented bioactive substances, and derived food ingredients. Especially, specialized 
ingredients in functional foods promoting the growth or activity of specific bacteria, such as Lactobacillus and Bifidobacterium genera, have been specifically examined as prebiotics. Recent scientific advances show that modifying the bacterial composition of the intestinal ecosystem induces functional changes such as the host physiology [16-19]. A diet with greater amounts of dietary fiber increases the composition of Clostridiales in mice. This is a dominant class of commensal microbes that produces butyric acid to induce colonic regulatory $\mathrm{T}$ (Treg) cells, which play a central role in suppressing inflammatory and allergic responses [20-22]. Therefore, it is crucially important to evaluate foods to ascertain whether they increase the composition of bacteria that produce bioactive molecules.

Fermented foods have been a fundamentally important part of the human diet for centuries in most parts of the world. Fermented foods of many types are produced from animal and plant materials, some of which have the potential to provide additional health benefits through fermentation as functional foods [23,24]. Some fermented foods have beneficial immune, glycemic, and anti-inflammatory activities [25-28], whereas other fermented foods increase some inflammation markers [29]. These results indicate the necessity for assessment of each fermented food to assess its health-promoting activities because of differences in the materials and microbes used for fermentation.

For this study, we used a fermented plant extract (FPE) produced from whole plants to examine the effects on dextran sulfate sodium (DSS)-induced colitis in a mouse model. Bloody stools and atrophy of colonic length were not observed. The TNF- $\alpha$ and IL- 6 concentrations were suppressed by FPE supplementation. Subsequently, 16S rRNA amplicon sequencing revealed that the composition of Clostridiales order had increased significantly in the fecal samples of young women after 3-month FPE supplementation with daily diet.

\section{Materials and Methods}

\subsection{Preparation of Fermented Plant Extract}

Whole fruits and vegetables with peels and seeds were minced and extracted using muscovado sugar in separate barrels (Table S1). For animal experimentation, the extracts were mixed with mushroom, pulse, cereals, seaweed, and decoction of loquat leaves, to make a starting material of 75 kinds, consisting of $50.0 \%$ of sugar, $23.9 \%$ of fruits, $15.6 \%$ of vegetables and wild herbs, $2.0 \%$ of mushrooms, $1.8 \%$ of seaweed, and $6.7 \%$ of pulse and cereals. Lactic acid bacteria, Lactiplantibacillus pentosus, L. plantaru, Pediococcus pentosaceus, P. acidilactici, Lacticaseibacillus paracasei, L. casei, Lactococcus lactis, Latilactobacillus curvatus, Leuconostoc mesenteroides, and Levilactobacillus brevis were added to the starting material and fermented at room temperature. After three year-fermentation and maturation, the fermented extract was strained and heated at $80^{\circ} \mathrm{C}$ for 10 min to obtain FPE. $\mathrm{pH}$, water content, and brix of were 3.8-4.8, 33-39\%, and 59-65\%, respectively. For microbiota analyses, the materials, which were acceptable for exporting to international countries, were mixed to make a starting material of 40 kinds, consisting of $42.0 \%$ of sugar, $24.6 \%$ of fruits, $19.4 \%$ of vegetables and wild herbs, $5.0 \%$ of mushrooms, $3.0 \%$ of seaweed, and $6.0 \%$ of pulse and cereals. The mixture was fermented using the same lactic acid bacteria and method as that described above. $\mathrm{pH}$, water content, and brix of the fermented extract were $3.8-4.5,34-42 \%$, and $57-62 \%$, respectively. As an FPE package, $5 \mathrm{~g}$ of the mixture, consisting of $40 \%$ of FPE, $59 \%$ of apple extract, and $1 \%$ of plum juice, was packed in an aluminum bag. The mixture was prepared to consist of $43.8 \%$ of apple juice, $26.3 \%$ of sugar, $29.0 \%$ of dextrin, and $0.9 \%$ of caramel color. Then, $5 \mathrm{~g}$ of the mixture was packed in an aluminum bag as a placebo package.

\subsection{Animals}

Male BALB/c mice were purchased from CLEA Japan, Inc. (Tokyo, Japan). They were kept under standard conditions in a room at $21-24^{\circ} \mathrm{C}$ with a constant $12 \mathrm{~h}$ light/dark cycle. The ethics committee of Sonoda Women's University approved the experiments, which were performed in accordance with relevant guidelines and regulations. 


\subsection{Induction of Colitis}

Five-week-old mice were divided into three groups: a negative group $(n=8)$, a positive group $(n=8)$, and an FPE group $(n=8)$. The positive group was fed diet with $3 \%$ dextran sulfated sodium 5000 (DSS) solution. The FPE group was fed diet containing 5\% FPE with 3\% DSS solution. The negative group was fed diet with water for 7 days. The diet composition provided to each group is presented in Table S2.

\subsection{IL- 6 and TNF- $\alpha$ Assays}

IL-6 and TNF- $\alpha$ concentrations in mouse sera were found using Mouse IL-6 Assay Kit (Immuno-Biological Laboratories Co., Ltd., Gunma, Japan) and Quantikine ELISA Mouse TNF- $\alpha$ Kit (R\&D Systems, Minneapolis, MN, USA), respectively, according to the instructions of the manufacturers.

\subsection{Dietary Supplementation and Fecal Collection}

We recruited 20 healthy women (age of 20-24 years, $21.4 \pm 1.2$ years) from the School of Human Sciences, Kobe College. All participants gave written, informed consent before participating in the study. This study was approved by the review board of Kobe College. Participants were assigned randomly into two groups. Participants in the FPE group were administered one FPE package three times after meals every day for 3 months in addition to their habitual diet. Participants in the placebo group were administered one placebo package in the same way as that of FPE group. No dietary restriction was instructed on participants. Participants collected fecal materials before and after 3-month administration. The materials were suspended in a solution (DNA/RNA Shield ${ }^{\mathrm{TM}}$; Zymo Research Corp., Irvine, CA, USA).

\subsection{Microbial DNA Extraction}

Total microbial DNA was extracted from each fecal suspension using a kit (QuickDNA $^{\mathrm{TM}}$ Fecal/Soil Microbe Miniprep; Zymo Research Corp., Irvine, CA, USA) according to the manufacturer's instruction. The DNA samples were examined using a spectrophotometer (Nanodrop ND-1000; Thermo Scientific, Waltham, MA, USA) by measuring absorbance values at 260 and $280 \mathrm{~nm}$.

\section{7. $16 S$ rRNA Gene Sequencing}

The V3-V4 region of the microbial 16S rRNA gene in each DNA sample was amplified by PCR using the universal primer set $341 \mathrm{~F} / 806 \mathrm{R}$ according to a protocol described elsewhere [30]. A dual-indexing amplification and sequencing approach was used. The resulting amplicons were purified and subjected to sequencing with $2 \times 301$ bp paired-end reads on the MiSeq systems (Illumina, Inc., San Diego, CA, USA) using MiSeq v3 reagent kit (Illumina) according the protocols described by Illumina (https:/ /jp.support.illumina.com/ content/dam/illumina-support/documents/documentation/chemistry_documentation/ 16s/16s-metagenomic-library-prep-guide-15044223-b.pdf, accessed on 5 April 2021). The raw sequence data quality was checked using the FastQC quality-control tool (Babraham Bioinformatics, Cambridge, United Kingdom; http:/ / www.bioinformatics.babraham.ac. $\mathrm{uk} /$ projects / fastqc/, accessed on 5 April 2021). The datasets were analyzed with QIIME 1.9.1 pipeline (Quantitative Insights Into Microbial Ecology; http: / qiime.org, accessed on 5 April 2021) using GreenGenes 13.5 [31].

\subsection{Statistical Analysis}

Data analysis was performed using the analysis of Bonferroni's multiple comparison test and Student's $t$-test, with a statistical significance set at $p<0.05$. 


\section{Results and Discussion}

\subsection{Anti-Colitis Effect of FPE in Mice}

Plant materials of fermented foods, such as asparagus, garlic, chicory, onion, wheat, barley, rye, soybean, peas, beans, banana, tomato, seaweeds, and microalgae, contain lactulose, galactooligosaccharides, fructooligosaccharides, inulin, maltoorogosaccharides, and resistant starch, known as "prebiotics", adversely affect the growth and activity of specific bacteria producing immunomodulatory products, particularly a short-chain fatty acid such as butyric acid. In addition, fermentation of these materials produces novel bioactive compounds working as prebiotics [25-28,32]. Therefore, we specifically examine the potential of plant materials and the produced FPE. FPE produced from extract of 75 kinds was rich in phytochemicals such as dietary fiber and polyphenol, and physiological function such as anti-oxidative, anti-inflammatory, and anti-allergy activities [33].

To assess the anti-colitis effects of FPE, acute colitis was induced by DSS feeding for 7 days to evaluate the effects of FPE on gut inflammation. The daily dietary intakes of negative group, positive group, and FPE group, which were, respectively, $2.1 \pm 0.2$, $1.8 \pm 0.2$, and $2.0 \pm 0.3 \mathrm{~g} /$ day, were not found to be significantly different. The positive group was found to have significantly reduced body weight after 7-day DSS administration $(21.9 \pm 0.31 \mathrm{~g})$ compared with results found for the negative group $(24.6 \pm 0.48 \mathrm{~g})$, although the FPE group was found to have no significant difference in body weight $(23.5 \pm 0.41 \mathrm{~g})$ compared with the negative group (Figure S1). The bloody stool appeared at 5 days after DSS administration in the positive group. Its color was brown. It progressed at 6 and 7 days. The color was red. In contrast, the bloody stool found in the FPE group was not observed as it was in the negative group, whereas the fecal color of the FPE group was black (Table 1). The colon length in the positive group $(5.9 \pm 0.17 \mathrm{~cm})$ was significantly shorter than that in the negative group $(8.3 \pm 0.24 \mathrm{~cm})$, but the FPE group exhibited no difference in the colon length, which was $7.7 \pm 0.21 \mathrm{~cm}$ compared with the negative group (Figure 1 and Figure S2). The concentrations of IL- 6 and TNF- $\alpha$, inflammatory cytokines, and markers of inflammation were increased significantly, five-fold and four-fold, respectively, compared with the negative group after 5-day DSS administration. However, those in the FPE group were not significantly different from those in the negative group (Figure 2). These results demonstrate that our FPE has an anti-inflammatory effect on DSS-induced mouse colitis.

Table 1. Blood stool score of feces.

\begin{tabular}{cccc}
\hline $\begin{array}{c}\text { Day after DSS } \\
\text { Administration }\end{array}$ & Negative Group & Positive Group & FPE Group \\
\hline 4 & - & - & $-(\mathrm{BK})$ \\
5 & - & + & $-(\mathrm{BK})$ \\
6 & - & ++ & $-(\mathrm{BK})$ \\
7 & - & ++ & $-(\mathrm{BK})$ \\
\hline
\end{tabular}

,- normal; + , brown bloody stool; ++ , red bloody stool; BK, black color.

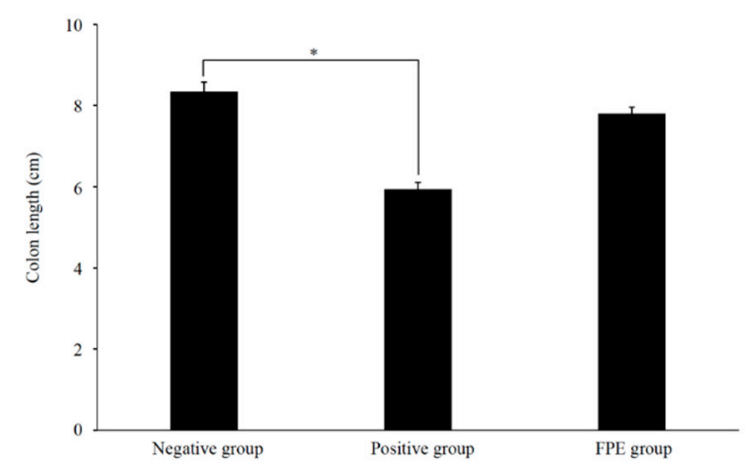

Figure 1. Colon length of mice after 7 day-administration. The values are expressed as means \pm SEM $(n=8) .{ }^{*} p<0.05$, accessed using Bonferroni's multiple comparison test. 

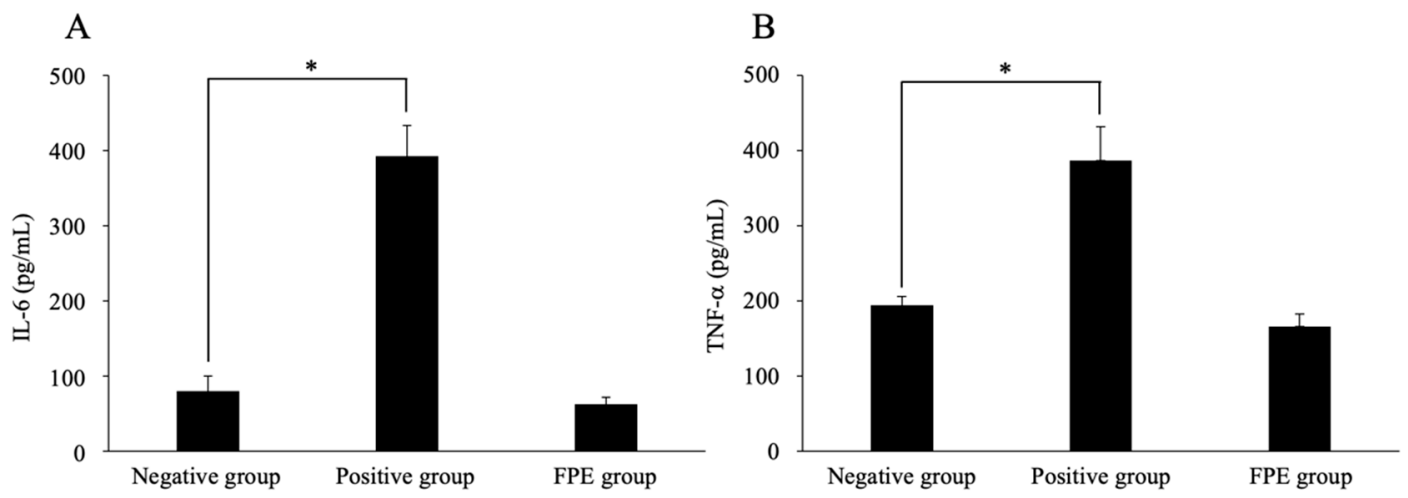

Figure 2. IL-6 (A) and TNF- $\alpha$ (B) concentrations of mice serum after 7-day administration. The values are expressed as means $\pm \operatorname{SEM}(n=8) .{ }^{*} p<0.05$, assessed using Bonferroni's multiple comparison test.

\subsection{Change of Gut Microbiota Composition in Human}

To investigate changes of gut microbiota composition attributable to dietary supplementation of FPE, FPE was produced from extracts of 40 kinds, of which plant materials were included in those of 75 kinds and total content of fruits, vegetables, and wild herbs, which are sources of prebiotic [34], was $44 \%$ in extract of 40 kinds and dietary fiber was 4.6 $\mathrm{g} / 100 \mathrm{~g}$ extract in FPE from extracts of 40 kinds, while total content of them in extract of 75 kinds was $39.5 \%$ and FPE from extract of 75 kinds contained $1.9 \mathrm{~g}$ dietary fiber $/ 100 \mathrm{~g}$ extract. FPE from extract of 40 kinds contained same kinds of nutritional compounds such as amino acid and organic acid as FPE from extract of 75 kinds (Table S3). Each of 20 participants supplemented one FPE package or placebo package was administered three times after meals every day for 3 months. The changes of body weight, body fat mass, muscle mass, and body mass index (BMI) of the FPE group after 3-month administration were not significantly different from those of the placebo group (Figure S3). Based on data collected through a questionnaire, the scores of health conditions of both the FPE group and the placebo group after 3-month administration were not significantly different compared with those before administration. These results indicate that FPE supplementation did not affect health or physical condition.

Microbial DNA was isolated from fecal samples collected from FPE and placebo groups before and after 3-month administration. The DNA sequencing of the V3-V4 amplicons from 40 samples revealed 5,295,873 paired-end sequence reads with an average of 132,397 reads per sample. After filtering to eliminate poor quality sequences, finally $4,890,551$ sequences with an average of 122,264 reads per sample were generated. The composition of bacterial phyla in feces of the FPE group before supplementation was not significantly different from that of the placebo group, of which Firmicutes was the most abundant, followed by Bacteroidetes and Actinobacteria. After 3 months of supplementation, the composition of Firmicutes in the FPE group increased along with reduction in Bacteroidetes. It was found to be significantly different from that in the placebo group $(p=0.001)$ (Table 2, Figure S4). Reportedly, dietary fiber can be designed to favor beneficial bacterial groups; certain Firmicutes access insoluble matrix fibers and resistant starch [35,36]. Plant materials in FPE, which contain fiber, starch, and oligosaccharides, can induce Firmicutes phyla in the gut. At the bacterial order level, the composition of Clostridiales, $39.0 \pm 1.8 \%$, increased significantly to $43.6 \pm 2.5 \%$ after 3 -month administration $(p=0.03)$ in the FPE group, whereas those in the placebo group before and after 3 months were $37.8 \pm 1.6 \%$ and $38.0 \pm 2.1 \%$, respectively (Figure 3, Table S4). Clostridiales order in FPE group after 3-month administration consisted of $49 \%$ of Lachnospiraceae family and 33\% of Ruminococcaceae family, which produce butyric acid [37,38]. Clostridiales induces colonic Treg cells and generates short-chain fatty acid, butyric acid, by fermentation of dietary fiber [39,40]. Butyric acid promotes to increase the expression of IL-10 in Treg cells and inhibits the activation of the transcription factor, NF- $\kappa \beta$, in gut cells. Increase of IL-10 in Treg cells 
and decrease of NF- $\mathrm{\beta} \beta$ lead the intestinal immune homeostasis, suppressing systemic and mucosal immune activation to control intestinal inflammation and contributing to maintaining tolerance towards gut microbiota [20-22,41]. Taken together, results show that FPE enhances Clostridiales growth in the gut and show that it has an anti-inflammatory effect on colitis.

Table 2. Microbiota composition at the phyla level in feces of placebo and fermented plant extract (FPE) groups before and after 3 month-administration (\%).

\begin{tabular}{ccccc}
\hline \multirow{2}{*}{ Phyla } & \multicolumn{2}{c}{ Placebo Group } & \multicolumn{2}{c}{ FPE Group } \\
\cline { 2 - 5 } & 0 Month & 3 Months & 0 Month & 3 Months \\
\hline Actinobacteria & $16.3 \pm 1.7$ & $15.8 \pm 1.7$ & $16.8 \pm 1.8$ & $15.5 \pm 1.1$ \\
Bacteroidetes & $36.1 \pm 1.3$ & $35.7 \pm 1.5$ & $32.2 \pm 2.6$ & $31.3 \pm 1.2$ \\
Cyanobacteria & 0 & 0 & $0.1 \pm 0.1$ & 0 \\
Firmicutes & $43.1 \pm 1.5$ & $42.6 \pm 2.1$ & $46.7 \pm 1.5$ & $50.2 \pm 1.5^{* *}$ \\
Fusobacteria & $0.2 \pm 0.1$ & $0.1 \pm 0.1$ & $0.2 \pm 0.1$ & $0.1 \pm 0.1$ \\
Lentisphaerae & 0 & $0.1 \pm 0.1$ & $0.3 \pm 0.2$ & 0 \\
Nitrospirae & 0 & 0 & 0 & 0 \\
Proteobacteria & $3.7 \pm 1.6$ & $2.2 \pm 0.4$ & $3.7 \pm 0.9$ & $2.7 \pm 0.5$ \\
Spirochaetes & 0 & 0 & 0 & 0 \\
Synergistetes & 0 & 0 & 0 & 0 \\
TM7 & 0 & 0 & 0 & 0 \\
Tenericutes & 0 & $0.3 \pm 0.3$ & 0 & 0 \\
Verrucomicrobia & $1.5 \pm 1.4$ & $3.2 \pm 2.1$ & 0 & 0 \\
\hline
\end{tabular}

** $p<0.01$, assessed using Student's t-test.

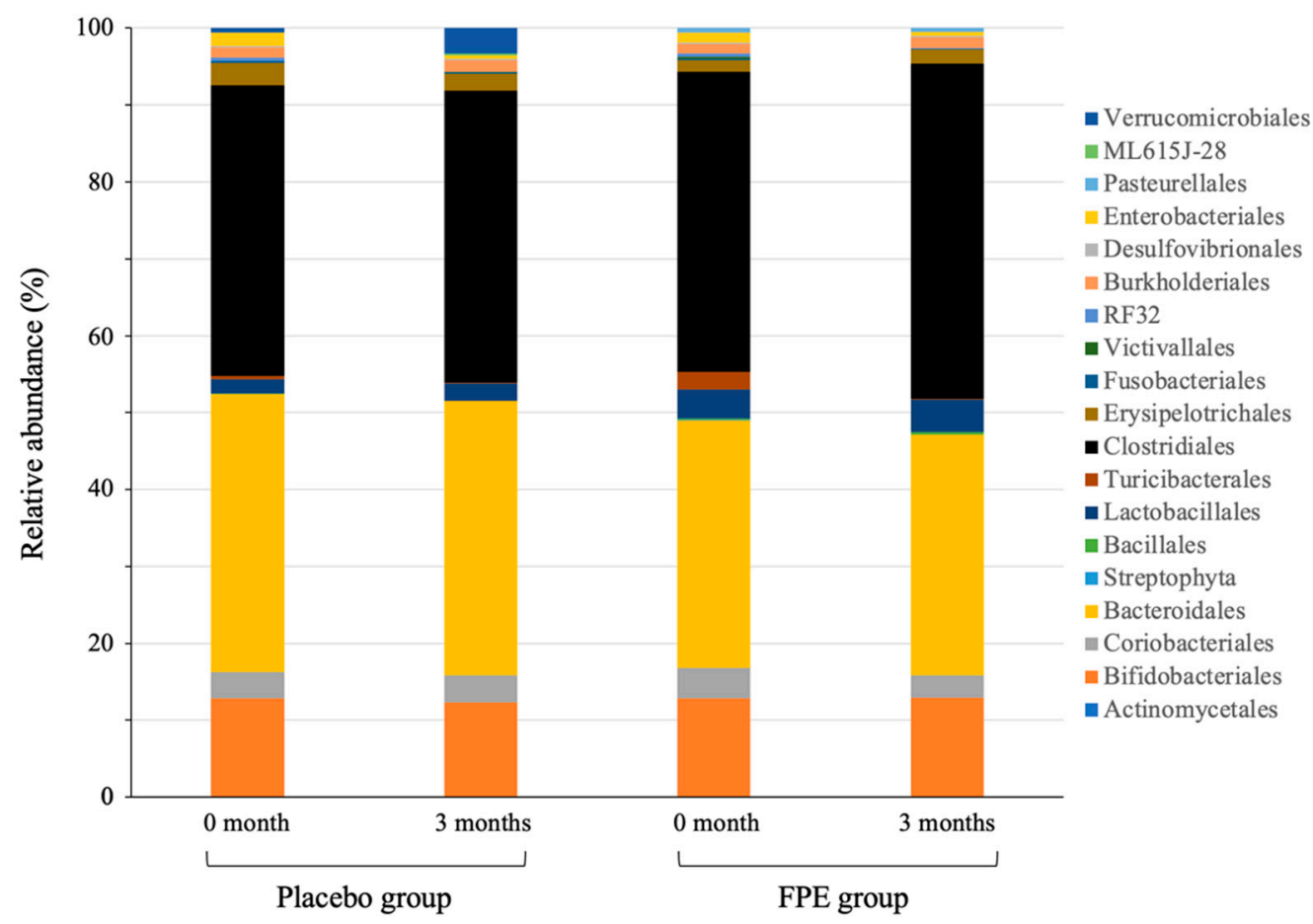

Figure 3. Microbiota composition at the order level in feces of placebo and FPE groups before and after 3-month administration.

Inflammatory bowel disease is generally treated with medicine. Lifestyle habits, particularly diet, have been implicated in the pathogenesis of inflammatory bowel disease [42]. Dietary therapy is assumed to lack life-threatening and severe side effects. However, a low-fat diet or a rich-fiber diet takes time and effort to prepare. Our study demonstrated that the daily diet supplemented with FPE directly prevented inflammation and changed the microbial composition in the gut, especially the increase of Clostridiales order, which 
plays a central role in suppressing inflammation, suggesting that FPE could improve the quality of human life against inflammatory bowel disease.

\section{Conclusions}

The daily diet was supplemented with FPE. Mice fed FPE with DSS for 7 days showed no body weight loss, atrophy of colonic length, or bloody stool. The concentrations of inflammation markers IL- 6 and TNF- $\alpha$ did not increase in the mice, demonstrating that FPE supplementation suppressed inflammation. The microbiota composition in feces from humans eating a diet supplemented with FPE for 3 months showed increased Clostridiales order, which produces short-chain fatty acids to suppress inflammation. These results indicate that FPE supplementation could enhance Clostridiales growth in the gut and indicate that it can have anti-inflammatory effects on colitis.

Supplementary Materials: The following are available online at https: / www.mdpi.com/article / 10.3390/fermentation7020055/s1. Table S1: Materials of FPE for animal and human. Table S2: Composition of experimental diet (\%). Table S3: Nutritional compound and amino acid content in FPE produced from 40 kinds of extracts. Table S4: Microbiota composition at the order level in feces of placebo and FPE groups before and after 3 month-administration (\%). Figure S1: Body weight of mice after 7-day administration. The values are expressed as means $\pm \operatorname{SEM}(n=8) .{ }^{*} p<0.05$, assessed using Bonferroni's multiple comparison test. Figure S2: Colon length of mice after 7-day administration. Figure S3: Change of body weight (A), fat mass (B), muscle mass (C), BMI (D), and health condition (E) of FPE group or placebo group. Values are expressed as means $\pm \mathrm{SD}(n=8)$ assessed using Student's t-test. Figure S3: Microbiota composition at the phyla level in feces of placebo and FPE groups before and after 3-month administration.

Author Contributions: M.S.: Conceptualization, Formal analysis, Investigation, Methodology, Writing—original draft, review and editing. T.W.: Conceptualization, Formal analysis, Investigation, Methodology, Writing - review and editing. M.T.: Conceptualization, Formal analysis, Investigation, Methodology, Writing — review and editing. K.S.: Formal analysis, Investigation, Methodology. T.M.: Resources, Writing-review and editing. N.M.: Resources, Writing-review and editing. S.S.: Resources, Methodology. All authors have read and agreed to the published version of the manuscript.

Funding: This research received no external funding.

Institutional Review Board Statement: Ethical review and approval were waived for this study. It was conducted according to legally established internal rules based on research ethics recommendations and with the informed consent of all study participants.

Informed Consent Statement: Informed consent was obtained from all study participants.

Data Availability Statement: The data described as a result of this study are available in the article and Supplementary Materials.

Conflicts of Interest: The authors declare that they have no conflict of interest.

\section{References}

1. Okabayashi, S.; Kobayashi, T.; Hibi, T. Inflammatory bowel disease in Japan-Is it similar to or different from Westerns? J. Anus Rectum Colon 2020, 4, 1-13. [CrossRef]

2. Japan Intractable Diseases Information Center. [Ulcerative Colitis (Designated Intractable Disease No. 97)]. Available online: https: / / www.nanbyou.or.jp/entry/62 (accessed on 5 April 2021). (In Japanese).

3. Bäckhed, F.; Ding, H.; Wang, T.; Hooper, L.V.; Koh, G.Y.; Nagy, A.; Semenkovich, C.F.; Gordon, J.I. The gut microbiota as an environmental factor that regulates fat storage. Proc. Natl. Acad. Sci. USA 2004, 101, 15718-15723. [CrossRef]

4. Wang, J.; Linnernbrink, M.; Künzel, S.; Fernandes, R.; Nadeau, M.J.; Rosenstiel, P.; Baines, J.F. Dietary history contributes to enterotype-like clustering and functional metagenomic content in the intestinal microbiome of wild mice. Proc. Natl. Acad. Sci. USA 2014, 111, 2703-2710. [CrossRef]

5. Willing, B.; Vörös, A.; Roos, S.; Jones, C.; Jansson, A.; Lindberg, J.E. Changes in faecal bacteria associated with concentrate and forage-only diets fed to horses in training. Equine Vet. J. 2009, 41, 908-914. [CrossRef]

6. Cani, P.D.; Bibiloni, R.; Knauf, C.; Waget, A.; Neyrinck, A.M.; Delzenne, N.M.; Burcelin, R. Changes in gut microbiota control metabolic endotoxemia-induced infammation in high-fat diet-induced obesity and diabetes in mice. Diabetes 2008, 57, 1470-1481. [CrossRef] 
7. Delzenne, N.M.; Cani, P.D. Gut microbiota and the pathogenesis of insulin resistance. Curr. Diab. Rep. 2011, 11, 154-159. [CrossRef]

8. Turnbaugh, P.J.; Hamady, M.; Yatsunenko, T.; Cantarel, B.L.; Duncan, C.A.; Ley, R.E.; Sogin, M.L.; Jones, W.J.; Roe, B.A.; Affourtit, J.P.; et al. A core gut microbiome in obese and lean twins. Nature 2009, 457, 480-484. [CrossRef]

9. Belkaid, Y.; Hand, T.W. Role of the microbiota in immunity and infammation. Cell 2014, 157, 121-141. [CrossRef]

10. Cani, P.D.; Osto, M.; Geurts, L.; Everard, A. Involvement of gut microbiota in the development of low-grade inflammation and type 2 diabetes associated with obesity. Gut Microbes 2012, 3, 279-288. [CrossRef]

11. Cani, P.D.; Amar, J.; Iglesias, M.A.; Poggi, M.; Knauf, C.; Bastelica, D.; Neyrinck, A.M.; Fava, F.; Tuohy, K.M.; Chabo, C.; et al. Metabolic endotoxemia initiates obesity and insulin resistance. Diabetes 2007, 56, 1761-1772. [CrossRef]

12. Fecteau, M.E.; Pitta, D.; Vecchiarelli, B.; Indugu, N.; Kumar, S.; Gallagher, S.C.; Fyock, T.L.; Sweeney, R.W. Dysbiosis of the Fecal Microbiota in Cattle Infected with Mycobacterium avium subsp. paratuberculosis. PLoS ONE 2016, 11, e0160353.

13. Ley, R.E.; Bäckhed, R.; Turnbaugh, P.; Lozupone, C.A.; Knight, R.D.; Gordon, J.I. Obesity alters gut microbial ecology. Proc. Natl. Acad. Sci. USA 2005, 102, 11070-11075. [CrossRef] [PubMed]

14. Sommer, F.; Backhed, F. The gut microbiota-Masters of host development and physiology. Nat. Rev. Microbiol. 2013, 11, 227-238. [CrossRef] [PubMed]

15. Grajek, W.; Olejnik, A.; Sip, A. Probiotics, prebiotics and antioxidants as functional foods: A review. Acta Biochim. Pol. 2005, 52, 665-671. [CrossRef]

16. Backhed, F.; Ley, R.E.; Sonnenburg, J.L.; Peterson, D.A.; Gordon, J.I. Host-bacterial mutualism in the human intestine. Science 2005, 307, 1915-1920. [CrossRef]

17. Manson, J.M.; Rauch, M.; Gilmore, M.S. The commensal microbiology of the gastrointestinal tract. Adv. Exp. Med. Biol. 2008, $635,15-28$.

18. McCracken, V.J.; Lorenz, R.G. The gastrointestinal ecosystem: A precarious alliance among epithelium, immunity and microbiota. Cell Microbiol. 2001, 3, 1-11. [CrossRef]

19. Lievin-Le Moal, V.; Servin, A.L. The front line of enteric host defense against unwelcome intrusion of harmful microorganisms: Mucins, antimicrobial peptides, and microbiota. Clin. Microbiol. Rev. 2006, 19, 315-337. [CrossRef]

20. Furusawa, F.; Obata, Y.; Fukuda, S.; Endo, T.A.; Nakato, G.; Takahashi, D.; Nakanishi, Y.; Uetake, C.; Kato, K.; Kato, T.; et al. Commensal microbe-derived butyrate induces the differentiation of colonic regulatory T cells. Nature 2013, 504, 446-450. [CrossRef]

21. Atarashi, K.; Tanoue, T.; Oshima, K.; Suda, W.; Nagano, Y.; Nishikawa, H.; Fukuda, S.; Saito, T.; Narushima, S.; Hase, K.; et al. Treg induction by a rationally selected mixture of Clostridia strains from the human microbiota. Nature 2013, 500, 232-236. [CrossRef]

22. Atarashi, K.; Tanoue, T.; Shima, T.; Imaoka, A.; Kuwahara, T.; Momose, Y.; Cheng, G.; Yamasaki, S.; Saito, T.; Ohba, Y.; et al. Induction of colonic regulatory $\mathrm{T}$ cells by indigenous Clostridium species. Science 2011, 331, 337-341. [CrossRef]

23. Marco, M.L.; Heeney, D.; Binda, S.; Cifelli, C.J.; Cotter, P.D.; Foligné, B.; Ganzle, M.; Kort, R.; Pasin, G.; Pihlanto, A.; et al. Health benefits of fermented foods: Microbiota and beyond. Curr. Opin. Biotechnol. 2017, 44, 94-102. [CrossRef] [PubMed]

24. Bell, V.; Ferrao, J.; Fernandes, T. Nutritional guidelines and fermented food frameworks. Foods 2017, 6, 65. [CrossRef]

25. Moussa, L.; Bézirard, V.; Salvador-Cartier, C.; Bacquié, V.; Lencina, C.; Lévêque, M.; Braniste, V.; Ménard, S.; Théodorou, V.; Houdeau, E. A low dose of fermented soy germ alleviates gut barrier injury, hyperalgesia and faecal protease activity in a rat model of inflammatory bowel disease. PLOS ONE 2012, 7, e49547. [CrossRef]

26. Bondia-Pons, I.; Nordlund, E.; Mattila, I.; Katina, K.; Aura, A.M.; Kolehmainen, M.; Orešič, M.; Mykkänen, H.; Poutanen, K. Postprandial differences in the plasma metabolome of healthy Finnish subjects after intake of a sourdough fermented endosperm rye bread versus white wheat bread. Nutr. J. 2011, 10, 116. [CrossRef]

27. Mueller, T.; Voigt, W. Fermented wheat germ extract-Nutritional supplement or anticancer drug? Nutr. J. 2011, 10, 89. [CrossRef] [PubMed]

28. Ryan, E.P.; Heuberger, A.L.; Weir, T.L.; Barnett, B.; Broeckling, C.D.; Prenni, J.E. Rice bran fermented with Saccharomyces boulardii generates novel metabolite profiles with bioactivity. J. Agric. Food. Chem. 2011, 59, 1862-1870. [CrossRef] [PubMed]

29. Ibrahim, F.; Ruvio, S.; Granlund, L.; Salminen, S.; Viitanen, M.; Ouwehand, A.C. Probiotics and immunosenescence: Cheese as a carrier. FEMS Immunol. Med. Microbiol. 2010, 59, 53-59. [CrossRef] [PubMed]

30. Caporaso, J.G.; Lauber, C.L.; Walters, W.A.; Berg-Lyons, D.; Lozupone, C.A.; Turnbaugh, P.J.; Fierer, N.; Knight, R. Global patterns of 16S rRNA diversity at a depth of millions of sequences per sample. Proc. Natl. Acad. Sci. USA 2011, 108, 4516-4522. [CrossRef] [PubMed]

31. Ariefdjohan, M.W.; Savaiano, D.A.; Nakatsu, C.H. Comparison of DNA extraction kits for PCR-DGGE analysis of human intestinal microbial communities from fecal specimens. Nutr. J. 2010, 9, 23. [CrossRef]

32. Davani-Davari, D.; Negahdaripour, M.; Karimzadeh, I.; Seifan, M.; Mohkam, M.; Masoumi, S.J.; Berenjian, A.; Ghasemi, Y. Prebiotics: Definition, types, sources, mechanisms, and clinical applications. Foods 2019, 8, 92. [CrossRef] [PubMed]

33. Kuwaki, S.; Nakajima, N.; Tanaka, H. Plant-based paste fermented by lactic acid bacteria and yeast: Functional analysis and possibility of application to functional foods. Biochem. Insights 2012, 5, 21-29. [CrossRef]

34. Al-Sheraji, S.H.; Ismail, A.; Manap, M.Y.; Mustafa, S.; Yusof, R.M.; Hassan, F.A. Prebiotics as functional foods: A review. J. Funct. Foods 2013, 5, 1542-1553. [CrossRef] 
35. Ze, X.; David, Y.B.; Laverde-Gomez, J.A.; Dassa, B.; Sheridan, P.O.; Duncan, S.H.; Louis, P.; Henrissat, B.; Juge, N.; Koropatkin, N.M.; et al. Unique organization of extracellular amylases into amylosomes in the resistant starch-utilizing human colonic Firmicutes bacterium Ruinococcus Bromii. mBio 2015, 6, e01058-15. [CrossRef]

36. Kaur, A.; Chen, T.; Green, S.J.; Mutlu, E.; Martin, B.R.; Rumpagaporn, P.; Patterson, J.A.; Keshavarzian, A.; Hamaker, B.R. Physical inaccessibility of a resistant starch shifts mouse gut microbiota to butyrogenic Firmicutes. Mol. Nutr. Food Res. 2019, 63, e1801012. [CrossRef]

37. Flint, H.J.; Duncan, S.H.; Scott, K.P.; Louis, P. Links between diet, gut microbiota composition and gut metabolism. Proc. Nutl. Soc. 2015, 74, 13-22. [CrossRef]

38. Vital, M.; Karch, A.; Pieper, D.H. Colonic butyrate-producing communities in humans: An overview using omics data. $m$ Systems 2017, 2, e00130-17. [CrossRef]

39. Kanauchi, O.; Andoh, A.; Iwanaga, T.; Fujiyama, T.; Mitsuyama, K.; Toyonaga, A.; Bamba, T. Germinated barley foodstuffs attenuate colonic mucosal damage and mucosal nuclear factor kappa B activity in a spontaneous colitis model. J. Gastroenterol. 1999, 14, 1173-1179. [CrossRef]

40. Andoh, A.; Bamba, T.; Sasaki, M. Physiological and anti-inflammatory roles of dietary fiber and butyrate in intestinal functions. J. Parenter. Enteral. Nutr. 1999, 23, S70-S73. [CrossRef]

41. Lopetuso, L.R.; Scaldaferri, F.; Petito, V.; Gasbarrini, A. Commensal Clostridia: Leading players in the maintenance of gut homeostasis. Gut Phathogens 2013, 5, 23. [CrossRef]

42. Levi, A.J. Diet in the management of Crohn's disease. Gut 1985, 26, 985-988. [CrossRef] [PubMed] 